\title{
Sagen, was ist?!
}

"Was ist Wahrheit? (Joh 18,38) Man mag sich vorstellen, dass Pilatus' Frage an Jesus im Johannesevangelium einen bestimmten Ton hatte: Was ist schon Wahrheit?

Was wahr ist, ist nach der englischen Redewendung ein matter of fact. Wir vertrauen darauf - durchaus nicht unkritisch -, dass andere Menschen jenes Wahre sagen, zu dem sie durch sorgfältiges Unterscheiden, Interpretieren und Prüfen gekommen sind; dass sie sagen, was ist. Nach Hannah Arendt hat es die Wahrheit darum schwer im öffentlichen Diskurs, denn "es gibt kein sicheres Erkennungszeichen, das Wahrheit von Meinung unterscheide ${ }^{1}{ }^{1}$ Meinungen sind nicht einfach Unwahrheiten, sondern Ausdruck der Mannigfaltigkeit, in der den Menschen die Welt erscheint. Dennoch ist das Verhältnis von Wahrheit und Meinung nur schwer zu bestimmen: Nicht jede erdachte Vernunftwahrheit, die als Meinung ausgegeben wird, muss auch einer Tatsachenwahrheit entsprechen; beiden liegt ein unterschiedlicher Wahrheitsanspruch zugrunde. Der öffentliche Raum, in dem Menschen ihre Meinungen austauschen, ist dabei notwendig und wesentlich plural. Durch den Austausch der Meinungen mittels öffentlicher Rede und Gegenrede können Menschen voreinander glänzen. Arendt weist darauf hin, dass das griechische Wort für die Meinung, die man hat, dóxa, gleichzeitig auch "Glanz« oder "Ruhm" bedeuten kann, im Sinne der Meinung, in der man bei anderen steht.

Dabei ist vorausgesetzt, dass die Menschen nicht nur sagen, was ist, sondern auch sagen, was sie tatsächlich meinen - dass sie wahrhaftig reden. Dietrich Bonhoeffer schreibt polemisierend: "Es gibt Menschen, die sich dazu berufen fühlen, jedem, der ihnen in den Weg kommt, >die Wahrheit zu sagen`, wie sie es ausdrücken. ${ }^{2}$ Wahrhaftigkeit aber braucht Mut. Es braucht den Mut, sorgfaltig $\mathrm{zu}$ unterscheiden, $\mathrm{zu}$ interpretieren und zu prüfen: Was ist meine Meinung und wie begründe ich sie? Es braucht auch den Mut, den anderen zu fragen: Was ist Deine Meinung und wie kommst Du dazu? Wahrhaftigkeit ist der eigentliche Massstab, an dem Diskurse gemessen werden. Wahrhaftigkeit gründet auf gegenseitigem Vertrauen und (politischer) Freundschaft. Nicht nur durch die Einflüsse der griechischen Philosophie, von denen Philosophinnen wie Arendt

1 Hannah Arendt, Sokrates. Apologie der Pluralität, eingel. v. Matthias Bormuth u. m. Erinnerungen v. Jerome Kohn, Berlin 2016, 34-85, hier 45.

2 Dietrich Bonhoeffer, Was heißt die Wahrheit sagen?, in: Konspiration und Haft 1940-1945, Dietrich Bonhoeffer Werke (DBW), Bd. 16, Gütersloh 1996, 619-629. 
geprägt sind, sondern auch aus der jüdisch-christlichen Tradition wissen wir, welche Bedeutung die Wahrhaftigkeit für ein Gemeinwesen hat (Ex 20,16; Eph 4,25). Im Gespräch mit dem Islam lernen wir, dass der Tugend der Wahrhaftigkeit im Qur'ān ebenso grosser Wert beigemessen wird (Sure 2,42).

Der katholische Theologe Hans Küng hat 1968 dem 20. Jahrhundert einen "Pathos der Wahrhaftigkeit» bescheinigt. Vielleicht mit Blick auf die »68er«, die wissen wollten, was war, war Küng überzeugt, dass die Menschen einen "Sinn für Aufrichtigkeit, Ehrlichkeit, Ursprünglichkeit, Echtheit, Wahrhaftigkeit im weitesten Sinne des Wortes « hätten. ${ }^{3}$ Vielleicht wusste er aber auch mit Bonhoeffer, »daß >die Wahrheit sagen je nach dem Ort, an dem man sich befindet, etwas verschiedenes bedeutet.$^{4}$ Küng hat, durchaus idealtypisch, ein wahrhaftiges Ringen um die Wahrheit im Blick. Er geht davon aus, dass sich die Menschen ihre Wahrheit(en) in der gesellschaftlichen Debatte oder im persönlichen Gespräch auf der Grundlage gegenseitigen Vertrauens sagen und den Ausgleich, den Konsens suchen. Dieses Ringen scheint charakteristisch für die Wahrhaftigkeit zu sein, sowohl im öffentlichen Diskurs als auch in der inneren Haltung des Menschen (zu sich selbst).

Heute werden offensichtliche Unwahrheiten als "alternative facts $\|^{5}$ präsentiert (so die Beraterin des US-amerikanischen Präsidenten Donald Trump, Kellyanne Conway). Wahrheit ist zu einem matter of belief and emotions geworden. Sind wir des Unterscheidens, Prüfens und Interpretierens müde geworden? Hatte der US-amerikanische Autor Ralph J. Keyes also Recht, als er bereits 2004 sein populärpsychologisches Buch mit dem Titel The Post-truth Era überschrieb? Ist diese Gegenwartsanalyse treffend?

Eine deutsche Boulevardzeitung warb vor einigen Jahren mit dem Slogan: "Jede Wahrheit braucht einen Mutigen, der sie ausspricht." Die Hermeneutische Blätter 24|1 2018 stellen nicht zuerst die Wahrheitsfrage. Sie wollen vielmehr den Begriff und die Tugend der Wahrhaftigkeit vor den Herausforderungen der Gegenwart interdisziplinär in den Blick nehmen: In welchem Verhältnis stehen Wahrheit und Wahrhaftigkeit? Was heisst »die Wahrheit sagen«? Was ist Wahrhaftigkeit? Wer ist wahrhaftig und wer ist es nicht? Gott? Der Mensch? Die Kirche? Wie bestimmen Theologie und Philosophie das Verhältnis des Menschen zur Wahrheit? In welchem Zusammen-

\footnotetext{
3 Hans Küng, Wahrhaftigkeit, Freiburg, 1968, 28.

4 DBW 16, 620.

5 Vgl. NBC News, Meet the Press 22.01.2017. Abschrift, https://www.nbcnews. com/meet-the-press/meet-press-01-22-17-n710491 (14.11.2017).
} 
hang stehen Wahrhaftigkeit und Selbstverstehen? Wie wird das Verhältnis des Menschen zur Wahrheit oder Falschheit seiner Aussagen gedacht? Wie kann der öffentliche Diskurs wahrhaftig sein, wenn sich Wahrheit von Meinung nicht unterscheidet? Welche konkrete Bedeutung hat Wahrhaftigkeit in der Ethik oder im interreligiösen Dialog? Wie wird Wahrhaftigkeit im Journalismus, den Politik- oder in den Geschichtswissenschaften reflektiert? Wird wahrgenommen, dass die eigene Interpretation eines Sachverhalts auch den eigenen Zwecken und Interessen dient? Gibt es Zeugnisse in Literatur und Kunst, die sich mit der Wahrhaftigkeit des Menschen auseinandersetzen?

Die hier versammelten Beiträge versuchen auf die gestellten Fragen zu antworten, bieten Denkanstösse und werfen neue Fragen auf. Die Autorinnen und Autoren stellen sich somit mutig der Herausforderung, sich an der aktuellen Debatte zu beteiligen und klar zu positionieren. Sie legen dar, was sie meinen. Dafür danken wir Ihnen vielmals. Wir sind gewiss, dass die Hermeneutische Blätter 24|1 · 2018 mit ihrem Thema "Wahrhaftigkeit" eine wertvolle Ergänzung zu den einschlägigen Werken über Wahrheit und Lüge darstellen.

Ein herzlicher Dank geht last, but not least an Susanne Schenker, die sich redaktionell um das vorliegende Heft gekümmert hat, den Satz sowie die Einarbeitung aller Korrekturen übernommen hat und uns mit Rat und Tat unterstützt hat.

Florian Oepping und Dominik Weyl 